\title{
MYCN-targeting miRNAs are predominantly downregulated during MYCN-driven neuroblastoma tumor formation
}

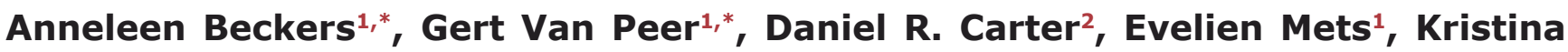 \\ Althoff $^{3,4}$, Belamy B. Cheung ${ }^{2}$, Johannes H. Schulte ${ }^{3,4,5,6}$, Pieter Mestdagh ${ }^{1}$, Jo \\ Vandesompele $^{1}$, Glenn M. Marshall ${ }^{2,7}$, Katleen De Preter ${ }^{1}$ and Frank Speleman ${ }^{1}$ \\ 1. Center for Medical Genetics (CMGG), Ghent University, Ghent, Belgium \\ 2. Children's Cancer Institute, University of New South Wales, Sydney, Australia \\ 3. Department of Pediatric Oncology and Hematology, University Children's Hospital Essen, Essen, Germany \\ 4. German Cancer Consortium (DKTK), Germany \\ 5. German Cancer Research Center (DKFZ), Heidelberg, Germany \\ 6. Translational Neuro-Oncology, West German Cancer Center, University Hospital Essen, University Duisburg-Essen, Essen, \\ Germany \\ 7. Kids Cancer Centre, Sydney Children's Hospital, Sydney, Australia \\ * These authors contributed equally to this work \\ Correspondence to: Frank Speleman, email: franki.speleman@ugent.be
}

Keywords: MYCN, microRNA, neuroblastoma, feedback regulation, cross-species

Received: July 01, $2014 \quad$ Accepted: September 15, $2014 \quad$ Published: September 16, 2014

This is an open-access article distributed under the terms of the Creative Commons Attribution License, which permits unrestricted use, distribution, and reproduction in any medium, provided the original author and source are credited.

\section{ABSTRACT}

MYCN is a transcription factor that plays key roles in both normal development and cancer. In neuroblastoma, MYCN acts as a major oncogenic driver through pleiotropic effects controlled by multiple protein encoding genes as well as microRNAs (miRNAs). MYCN activity is tightly regulated at the level of transcription and protein stability through various mechanisms. Like most genes, MYCN is further controlled by miRNAs, but the full complement of all miRNAs implicated in this process has not been determined through an unbiased approach. To elucidate the role of miRNAs in regulation of MYCN, we thus explored the MYCN-miRNA interactome to establish miRNAs controlling MYCN expression levels. We combined results from an unbiased and genome-wide high-throughput miRNA target reporter screen with miRNA and mRNA expression data from patients and a murine neuroblastoma progression model. We identified 29 miRNAs targeting MYCN, of which 12 miRNAs are inversely correlated with MYCN expression or activity in neuroblastoma tumor tissue. The majority of MYCN-targeting miRNAs in neuroblastoma showed a decrease in expression during murine MYCN-driven neuroblastoma tumor development. Therefore, we provide evidence that MYCN-targeting miRNAs are preferentially downregulated in MYCNdriven neuroblastoma, suggesting that MYCN negatively controls the expression of these miRNAs, to safeguard its expression.

\section{INTRODUCTION}

MYCN acts as a major driver oncogene in neuroblastoma, a pediatric tumor of the sympathetic nervous system. Genomic amplification of this gene occurs in about half of all high-stage neuroblastoma tumors and has been associated with poor survival. As such, MYCN amplification has been used as one of the first biomarkers for therapeutic decision-making.

MYC proto-oncogenes (MYC, MYCN, MYCL) are transcription factors that play key roles in both normal development and cancer. The MYC proteins act as direct amplifiers of transcriptionally active genes through sequence-specific binding to consensus E-box DNA binding sites [1-3]. In addition, MYC proteins can also silence genes, by a mechanism that is uncoupled from 
E-box binding and dependent upon initiator elements in the basal promoter region $[1,4]$. Apart from the broad impact on transcriptional regulation of protein coding genes, MYCN has also been shown to tightly control the expression of many microRNAs (miRNAs) [1,5].

Numerous signal transduction pathways and regulatory mechanisms keep the expression of MYC family members under tight control. This regulation occurs at multiple levels, including gene transcription, messenger RNA (mRNA) turnover, and protein activity and stability [1-3]. miRNAs have also been shown to contribute to the regulation of MYC expression [2,3]. miRNAs are involved in the regulation of virtually all signaling circuits within a cell, and their dysregulation has been shown to play an essential role in the development and progression of cancer [6,7]. This class of small noncoding RNA molecules typically inhibits the translation and stability of mRNA molecules and thus eventually controls protein levels.

In previous studies, individual miRNAs targeting MYCN have been identified, using miRNA target reporter assays to confirm predicted miRNA target sites in the 3' untranslated region (3'UTR) of MYCN [3,8]. Eleven MYCN-targeting miRNAs have thus been identified, of which miR-34a, miR-101, let-7e and miR-202 were shown to affect neuroblastoma proliferation in vitro $[3,8]$. Although the applied approach is valuable, it is biased towards canonical miRNA-mRNA interactions, identified by available prediction algorithms.

Here, we performed a comprehensive, genomewide exploration of the miRNA-MYCN interactome in neuroblastoma. We combined results from an unbiased and genome-wide high-throughput miRNA target reporter screen with miRNA and mRNA expression

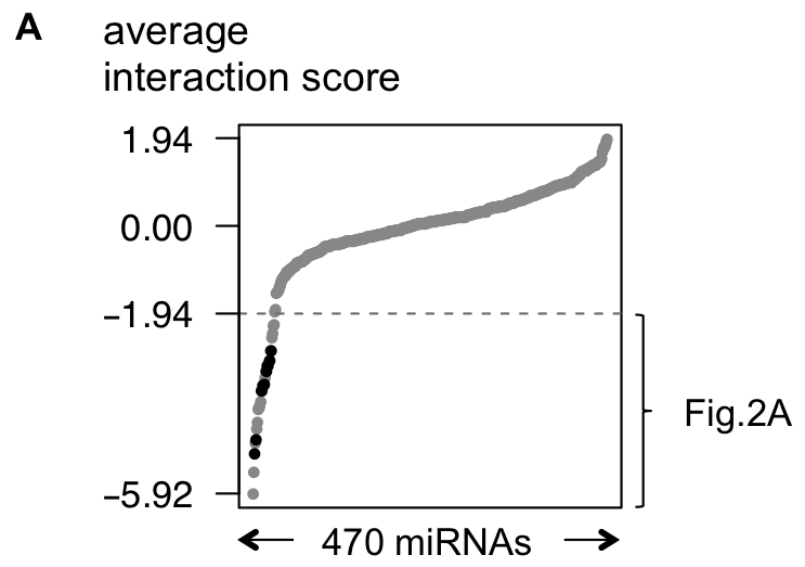

data from patients and identified 12 MYCN-targeting miRNAs in neuroblastoma tumor tissue. Subsequently, the dynamic regulation of MYCN-targeting miRNAs during neuroblastoma development was evaluated in a murine neuroblastoma progression model. We provide evidence that MYCN targeting miRNAs are preferentially downregulated in MYCN-driven neuroblastoma tumors, suggesting that MYCN negatively controls the expression of these miRNAs, and as such safeguards its own expression. Hence, our findings further clarify the role of miRNAs in the regulation of MYCN in neuroblastoma and describe a negative feedback loop from MYCN to its targeting miRNAs.

\section{RESULTS}

\section{An unbiased MYCN 3'UTR-miRNA library screen identifies 29 miRNAs targeting MYCN}

Potential interactions of 470 miRNAs with the 3'UTR of MYCN were assayed in a high-throughput luciferase reporter screen. In brief, human embryonic kidney cells (HEK293T) were co-transfected with a reporter construct, containing the MYCN 3'UTR downstream of a luciferase reporter gene, and each of the individual miRNA mimics from a 470 miRNA mimic library. Based on the relative luciferase activities in two independent screens (Supplementary Fig. S1), an interaction score was calculated for each miRNA-MYCN combination (see Material and Methods). This effort was part of a large-scale 3'UTR screening in which the interactions of 470 miRNAs with 17 selected cancer- and

\section{B cumulative fraction}

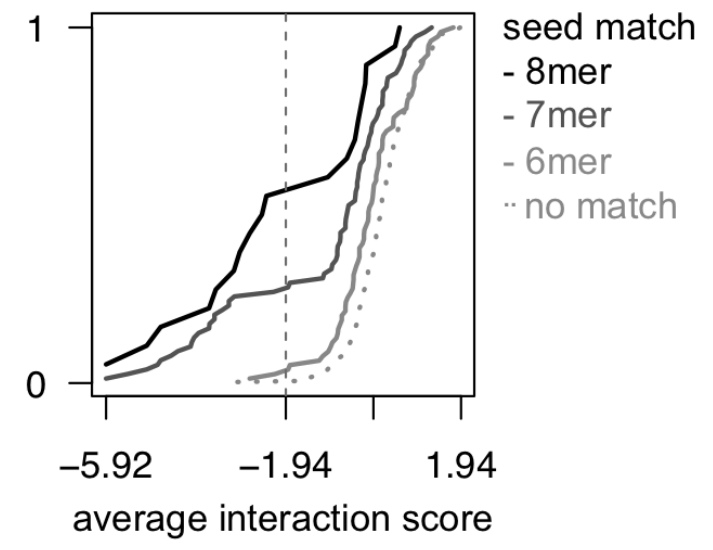

Figure 1: An unbiased MYCN 3'UTR-miRNA library screen identifies 29 miRNAs potentially targeting MYCN. (A) Average interaction scores are plotted (Y-axis) for 470 tested miRNAs (X-axis). The 29 miRNAs with an interactions core of $<-1.94$ are listed in Fig. 2A. The 11 interactions that have been reported in literature, are indicated in black. (B) Cumulative distributions (Y-axis) of the interaction scores (X-axis) of miRNAs that respectively have 6-, 7- or 8-mer seed-matches in the MYCN 3'UTR. 
disease-associated genes were probed (Van Peer et al., in preparation).

Applying this strategy, we identified 29 miRNAs with a high probability of targeting MYCN (interaction score $<-1.94$; see Material and Methods; Fig. 1A, Fig. 2A and Supplementary Table S6). All 11 previously established miRNA-MYCN interactions [3,8] were validated in our screen (Fig. 1A). In the same studies, 9 miRNAs predicted to target MYCN could not be validated; this is now confirmed by our data. Additionally, 18 new MYCN targeting miRNAs were identified, of which only 2 are predicted to target MYCN by MirTarget2, underscoring the value of this screening method to detect novel, predicted as well as non-predicted, miRNA-target
A

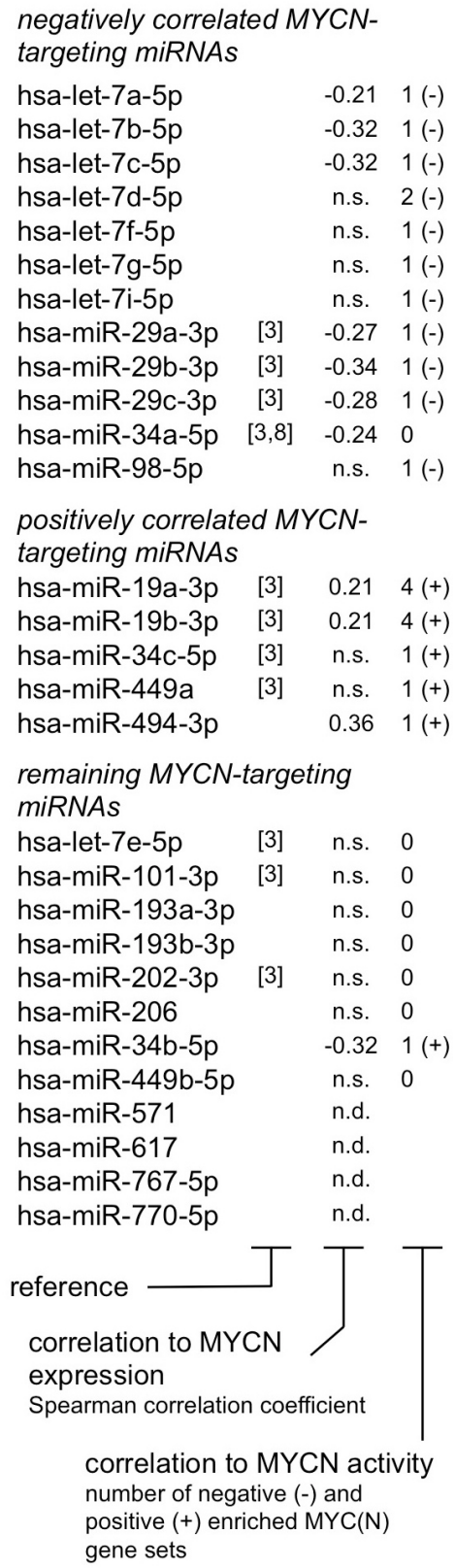

B cumulative fraction

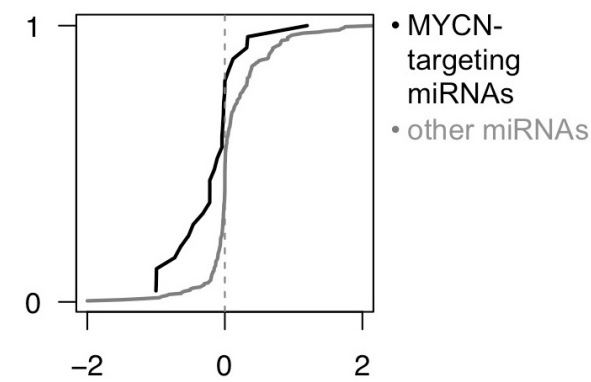

$\pi$-value, Spearman correlation

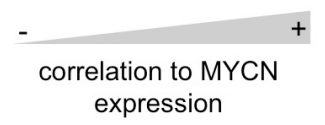

C negative enrichment of MYC $(\mathrm{N})$ gene sets

\begin{tabular}{|c|c|c|}
\hline & yes & no \\
\hline MYCN-targeting miRNAs & 11 & 14 \\
\hline \multirow[t]{2}{*}{ other miRNAs } & 64 & 188 \\
\hline & & 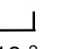 \\
\hline
\end{tabular}

Figure 2: Integration of 3'UTR-miRNA library screen and patient expression data identifies 12 MYCN-targeting miRNAs in neuroblastoma. (A) MYCN targeting miRNAs are filtered for their relevance in neuroblastoma according to two criteria: significant negative correlation to MYCN expression in a MYCN non-amplified neuroblastoma patient cohort and negative enrichment for MYC(N) gene sets, using Gene Set Enrichment Analysis. 12/29 miRNAs match with at least one of these criteria and are considered to be relevant for neuroblastoma. n.s.: not significant (Spearman correlation, Benjamini \& Hochberg multiple testing corrected p-value $>0.05$ ); n.d.: no data available. (B) The cumulative distribution (Y-axis) of the $\pi$-value for the correlation between miRNA and MYCN expression (X-axis) of miRNAs that were identified as either MYCN-targeting (black) or not MYCN-targeting (gray). Kolmogorov-Smirnov test, $\mathrm{p}$-value $=1.4 \times 10^{-3}$. (C) $2 \times 2$ contingency table for negative enrichment of MYC(N) gene sets of MYCN-targeting and other miRNAs. Chi-Square test. 
gene interactions. The top 5 miRNAs (miR-449b-5p, miR-767-5p, miR-98-5p, let-7b-5p and let-7f-5p) not reported in literature were validated by demonstrating rescue of reporter gene downregulation upon mutation of potential binding sites (Van Peer et al., in preparation). Among the strongest hits in the screen, a significant enrichment was observed for both miRNAs with seedmatched sites present in the MYCN 3 'UTR (Fig. 1B) and MirTarget2 (Supplementary Fig. S2) predicted miRNAs, thus further underscoring the sensitivity and robustness of the screen.

\section{Integration of 3'UTR-miRNA library screen and patient expression data identifies $12 \mathrm{MYCN}$ - targeting miRNAs in neuroblastoma}

miRNAs are known to regulate their target genes in a highly tissue- and cell type specific manner [9]. As the MYCN 3'UTR-miRNA library screen was performed in HEK293T cells, we next aimed to specifically select miRNAs targeting MYCN in neuroblastoma cells by integrating the obtained screen results with miRNA expression data from primary neuroblastoma tumors. We reasoned that the expression of a targeting miRNA is inversely correlated to MYCN mRNA expression levels or

(B)

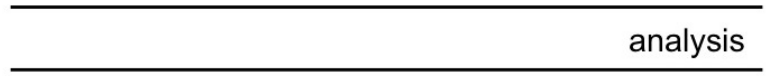

integration with expression data of primary tumors

(C)

analysis
TH-MYCN mouse model

LSL-MYCN;Dbh-iCre mouse model

SHEP-MYCN-ER cell line

(A)

MYCN 3'UTR screen
MYCN activity in primary tumor samples. Assuming that miRNAs would have more impact on MYCN expression levels and activities in MYCN non-amplified cells, we chose to assess miRNA correlations with MYCN mRNA levels and activity only in this tumor subset to infer a potential regulatory relationship.

\section{Correlation to MYCN mRNA expression}

Overall, MYCN targeting miRNAs showed stronger inverse correlation to MYCN mRNA levels compared to non-targeting miRNAs (Fig. 2B), as indicated by a shift of the cumulative distribution of $\pi$-values ( $-\log 10 \mathrm{p}$-value * Pearson correlation coefficient) to more negative values. Of the $25 \mathrm{MYCN}$-targeting miRNAs that were included on the human miRNA expression platform, 8 miRNAs showed statistically significant inverse correlation to MYCN mRNA expression, suggesting that they can indeed downregulate MYCN expression in primary MYCN non-amplified tumors. Three miRNAs were positively correlated to MYCN expression. Two of these miRNAs, miR-19a-3p and miR-19b-3p, are part of the oncogenic miR-17-92 cluster and were already known to be directly induced by MYCN in neuroblastoma cells [10].

\section{Correlation to MYCN activity}

miRNAs are known to regulate their targets by inducing mRNA destabilization and translational

\begin{tabular}{|c|c|c|c|}
\hline \multicolumn{2}{|c|}{ dataset } & \multicolumn{2}{|c|}{ results } \\
\hline included & missing & MYCN-targeting & not targeting \\
\hline 470 & - & (29) & 441 \\
\hline \multicolumn{4}{|c|}{$\sqrt{ }$} \\
\hline included & missing & inverse correlation & correlation \\
\hline 25 & 4 & (12) & \\
\hline \multicolumn{4}{|c|}{ 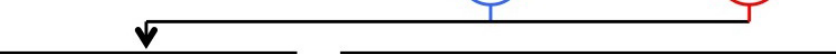 } \\
\hline included & missing & downregulated & upregulated \\
\hline 12 & 0 & $10 / 12$ & $0 / 12$ \\
\hline 5 & 0 & $1 / 5$ & $3 / 5$ \\
\hline 9 & 3 & $9 / 9$ & $0 / 9$ \\
\hline 5 & 0 & $1 / 5$ & $3 / 5$ \\
\hline 12 & 0 & $2 / 12$ & $0 / 12$ \\
\hline 5 & 0 & $0 / 5$ & $3 / 5$ \\
\hline
\end{tabular}

Figure 3: Overview of results from different analyses. Numbers listed represent the number of miRNAs. (A) Of 470 screened miRNAs, 29 miRNAs interact with the 3'UTR of MYCN. (B) Of these 29 miRNAs, 25 miRNAs could subsequently be analyzed in primary neuroblastoma tumors: 12 miRNAs are inversely correlated to MYCN expression or activity, whereas 5 miRNAs are positively correlated to MYCN expression or activity. (C) Of the 12 miRNAs inversely correlated to MYCN expression or activity, 10 miRNAs are downregulated during MYCN-driven tumor development in the TH-MYCN mouse model, 9 miRNAs are lower expressed in LSLMYCN;Dbh-iCre tumors compared to wild-type adrenals, and 2 miRNAs are repressed upon induction of MYCN in the SHEP-MYCN-ER model system. On the other hand, of the 5 miRNA correlated to MYCN expression or activity, 3 miRNAs are upregulated during MYCNdriven tumor development in the TH-MYCN mouse model, these miRNAs are also higher expressed in LSL-MYCN;Dbh-iCre tumors compared to wild-type adrenals, and 3 miRNAs are induced upon induction of MYCN in the SHEP-MYCN-ER model system. 
inhibition. Assessing the correlation between miRNA and MYCN mRNA levels, however, will not identify potential regulatory effects at protein level. Therefore, we additionally evaluated which miRNAs are inversely correlated to MYCN activity as a surrogate for MYCN protein levels. MYCN activity is reflected in the expression of MYCN regulated genes and can be assessed using Gene Set Enrichment Analysis (GSEA) [11]. In brief, for each miRNA, mRNAs were ranked according to decreasing expression correlation in MYCN nonamplified neuroblastoma tumors. Subsequently, GSEA was performed using six selected gene sets of MYC(N) upregulated genes [12-17] (see Materials and Methods). A miRNA negatively correlated to MYCN protein levels and thus activity - is expected to be negatively correlated to genes that are positively regulated by MYCN. In this case an enrichment of MYCN upregulated genes can be expected among the most negatively correlated genes at the bottom of the ranked list. Here, a miRNA was considered to be negatively correlated to MYCN activity in case such negative enrichment could be observed for at least one MYC(N) gene set (normalized enrichment score $<-2$ and false discovery rate $<0.25)$. As expected, the MYCN-targeting miRNAs identified in the 3'UTRmiRNA library screen were significantly enriched for miRNAs that showed negative enrichment for at least one MYC(N) gene set and thus for miRNAs that are negatively correlated to MYCN activity (Fig. 2C). Of the $25 \mathrm{MYCN}$ targeting miRNAs that were included on the human miRNA expression platform, 11 miRNAs showed this inverse relation to MYCN activity, whereas 6 miRNAs showed a positive relation to MYCN activity (Fig. 2A, Supplementary Table S7).

In summary, 12 of 29 MYCN-targeting miRNAs were inversely correlated to either MYCN expression or MYCN activity in MYCN non-amplified tumor samples, and therefore likely regulate $\mathrm{MYCN}$ in neuroblastoma (Fig 2A, Fig. 3). Interestingly, $5 \mathrm{MYCN}$-targeting miRNAs were positively correlated to MYCN expression or activity (Fig. 2A), suggesting that MYCN induces their expression in neuroblastoma. One miRNA, miR-34b-5p, shows both negative correlation to MYCN mRNA levels and positive correlation to MYCN activity (Fig. 2A) and is therefore excluded from further analyses.

\section{miRNAs that regulate MYCN are downregulated during murine MYCN-driven neuroblastoma tumor formation.}

In a next step, we explored how expression levels of miRNAs that target MYCN are dynamically regulated during MYCN-driven tumor formation. To this end, we performed miRNA expression profiling of the TH-MYCN mouse model, a murine MYCN-driven neuroblastoma progression model [18]. In brief, we sacrificed TH-MYCN+/+ mice one and two weeks after birth to harvest superior cervical and celiac ganglia containing foci of neuroblast hyperplasia, and 6-week old TH-MYCN+/+ mice to dissect advanced neuroblastoma tumors, arising from the neuroblast hyperplasia [19]. Additionally, we dissected the superior cervical and celiac ganglia from TH-MYCN-/- mice one, two and six weeks after birth to control for miRNA expression changes during normal postnatal development of the sympathetic ganglia. In the following analyses we only considered those murine miRNA assays that target sequences similar to the sequences of the human miRNAs included in our 3'UTR-miRNA library screen $(\mathrm{n}=187$, see Supplementary Table S6). To validate this MYCN-driven neuroblastoma progression model, we evaluated the expression of 50 known MYC(N)-regulated miRNAs [5], summarized in a MYCN signature score. This MYCN signature score increases significantly throughout tumor progression from tumor-prone ganglia to tumors in transgenic mice, compared to wild-type ganglia (Fig. 4A and Supplementary Fig. S3). This observation is in keeping with the hypothesis that sustained MYCN activity in the sympathetic ganglia of homozygous transgenic mice gives rise to the appearance of neuroblastoma tumors in these anatomic locations [19].

The majority of the MYCN-targeting miRNAs in neuroblastoma (10 out of 12) showed a significantly decreased expression during tumor development in TH-MYCN $+/+$ mice compared to normal development, while 2 of them showed no significantly different expression pattern (Fig. 3, Fig. 4B). Interestingly, of the 5 MYCN-targeting miRNAs with positive correlation to MYCN expression or activity, 3 miRNAs, miR-19a$3 p$, miR-19b-3p and miR-494-3p, showed significantly increased expression during tumor development (Fig. 4B), further supporting the hypothesis that MYCN induces the expression of these miRNAs. The 2 remaining positively correlated miRNAs, miR-34c-5p and miR-449a, are, respectively, significantly downregulated and not regulated during tumor development (Fig. 4B). Overall, the 12 MYCN-targeting miRNAs in neuroblastoma, show significantly more downregulation during MYCN-driven tumor development, compared to non-targeting miRNAs, as indicated by a shift of the cumulative distribution of the $\pi$-values (- $\log 10 p$-value $* \Delta$ slope) to more negative values (Fig. 4C).

The above findings could be confirmed in an additional, recently developed, MYCN-driven neuroblastoma mouse model, the LSL-MYCN;Dbh-iCre mouse model [20]. All 9 MYCN-targeting miRNAs that could be evaluated are significantly lower expressed in LSL-MYCN;Dbh-iCre tumors compared to wild-type adrenals (Fig. 3, Fig. 4D). Considering the $5 \mathrm{MYCN}-$ induced miRNAs, the data in the LSL-MYCN;Dbh-iCre tumors again fully recapitulated the findings from the TH-MYCN progression model: miR-19a-3p, miR-19b-3p 
and miR-494-3p showed increased expression in tumors compared to wild-type control tissue (Fig. 4D), whereas miR-34c-5p and miR-449a are respectively significantly downregulated and not regulated in LSL-MYCN;Dbh-iCre tumors.

\section{MYCN can directly modulate the expression levels of particular miRNAs that target its 3'UTR.}

In a final step, we explored to what extent MYCN directly controls, as transcriptional activator and repressor, the expression levels of the 12 selected MYCN-targeting

C cumulative fraction

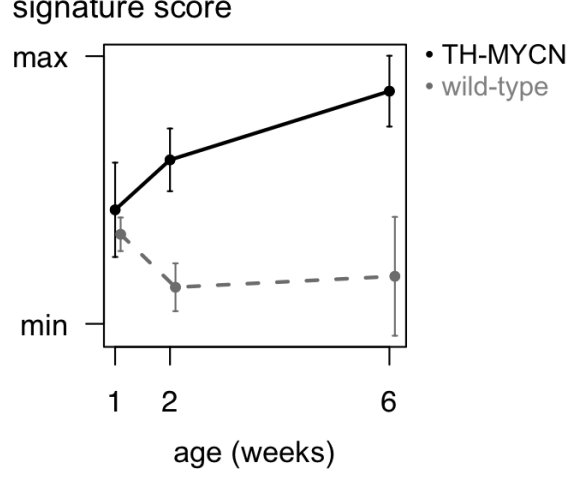

m-value, linear regression

\section{downregulation during tumor formation}

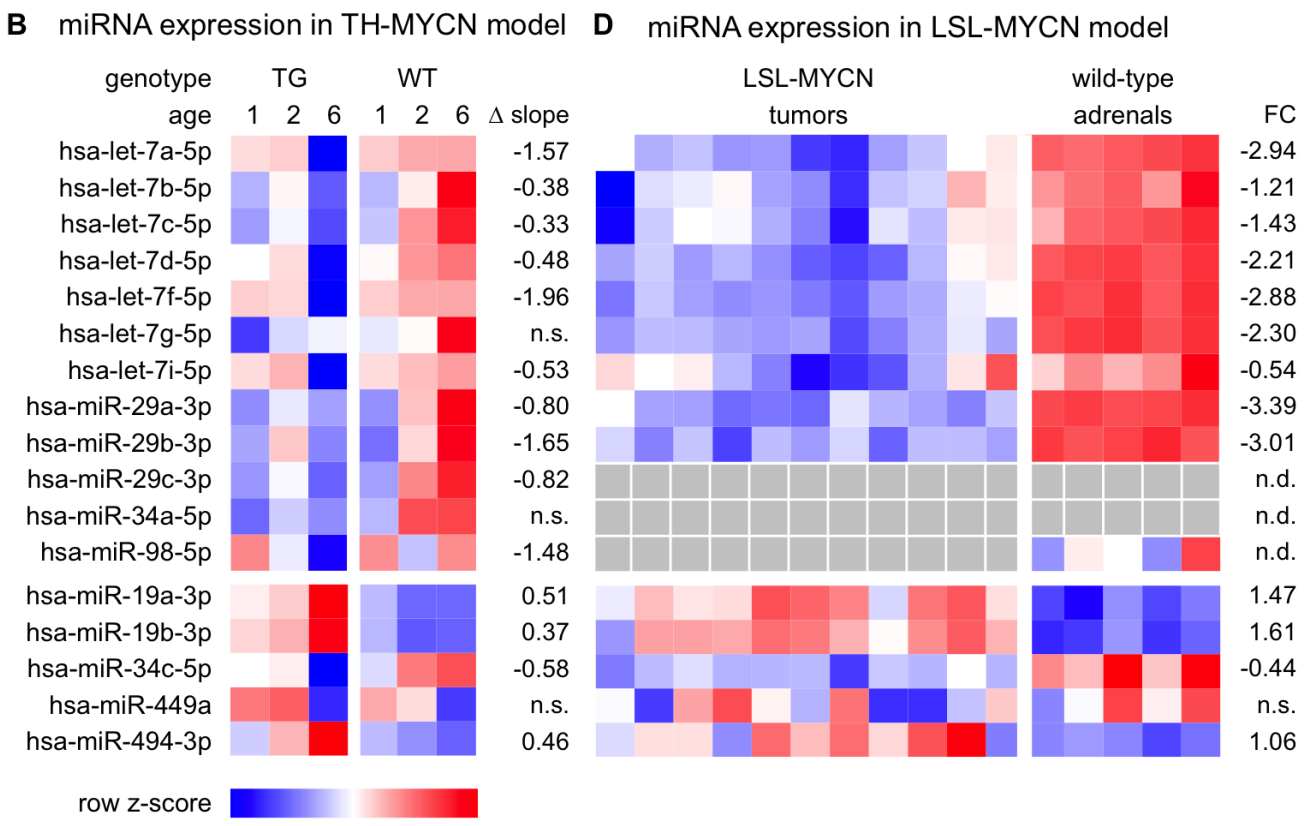

Figure 4: miRNAs that regulate MYCN are downregulated during murine MYCN-driven neuroblastoma tumor formation. (A) The MYCN miRNA signature score, representing expression of known MYC(N)-regulated miRNAs, increases during neuroblastoma progression in ganglia from TH-MYCN mice (TG, black) compared to ganglia from wild-type mice (WT, gray dashed). Data are presented as mean \pm standard deviation of four samples. (B) Heatmap of average miRNA expression levels during murine MYCNdriven neuroblastoma development. Data are presented as row normalized. TG: transgenic; WT: wild-type; $\Delta$ slope: the difference in regression slope between TG and WT samples; n.s.: non-significant difference (Benjamini \& Hochberg multiple testing corrected p-value $>0.05$ ). (C) The 12 MYCN-targeting miRNAs in neuroblastoma show significantly more downregulation during MYCN-driven tumor development, compared to non-targeting miRNAs. Kolmogorov-Smirnov test, p-value $=9.2 \times 10^{-3}$. (D) Heatmap of miRNA expression levels in 11 LSL-MYCN;Dbh-iCre tumors and 5 normal adrenals. For miR-29c-3p and -34a-5p, no expression data was available; miR-98$5 \mathrm{p}$ was not expressed in LSL-MYCN;Dbh-iCre tumors. Data are presented as row normalized. gray: no data available. FC: log2 fold change; n.d.: no data available; n.s.: non-significant fold change (Student T-test, Benjamini \& Hochberg multiple testing corrected p-value $>0.05$ ). 
miRNAs. To this end we measured miRNA expression levels in a MYCN-inducible model system. Two out of 12 MYCN-targeting miRNAs in neuroblastoma (Fig. 2A), miR-29a-3p and miR-29b-3p, showed significant reduction in expression $48 \mathrm{~h}$ after MYCN activation (Fig. 3, Fig. 5).

Of the five MYCN-targeting miRNAs with positive correlation to MYCN expression or activity (Fig. 2A), three miRNAs, miR-19b-3p, miR-19a-3p and miR-34c-5p, showed induction of expression (Fig. 5), supporting the assumption that MYCN induces the expression of these miRNAs.

\section{DISCUSSION}

Previous studies have highlighted the importance of miRNAs in the regulation of $\mathrm{MYCN}$, a major driver oncogene in neuroblastoma. Nonetheless, a comprehensive, genome-wide exploration of the miRNAMYCN interactome has not yet been performed. Thus far, studies have only focused on individual miRNAs or a small subset of miRNAs predicted to target MYCN by algorithms that are biased towards the limited knowledge of miRNA binding site architecture. Here, we report on the first unbiased and genome-wide high-throughput miRNA target reporter screen to identify miRNAs targeting MYCN. From the total of 29 MYCN-targeting miRNAs identified in this study, 18 interactions were novel whereas all 11 previously established MYCN-targeting miRNAs $[3,8]$ were confirmed, thus underscoring the validity of our genome-wide high-throughput screen.

Previously identified MYCN-targeting miRNAs have not been confirmed to regulate MYCN in primary neuroblastoma tumor cells. To address this shortcoming, we integrated our screen results with matching miRNA and mRNA expression profiles from primary neuroblastoma tumors. For 12 MYCN-targeting miRNAs we observed significant inverse correlation to $\mathrm{MYCN}$ expression or activity and thus provide evidence that they regulate MYCN in a neuroblastoma tissue context: eight let-7 family miRNAs (let-7a-5p, let-7b-5p, let-7c-5p, let-7d-5p, let-7f-5p, let-7g-5p, let-7i-5p and miR-98), three miR-29 family miRNAs (miR-29a-3p, miR-29b-3p, miR-29c-3p) and miR-34a-5p.

In a next step, we investigated the dynamic regulation of these miRNAs during the process of MYCN-driven tumor formation, using the extensively validated TH-MYCN mouse model. Notably, we observe that MYCN-targeting miRNAs are preferentially downregulated during MYCN-driven tumor development, suggesting that MYCN negatively regulates the miRNAs by which it is targeted, to safeguard its own expression. Growing evidence suggests that MYCN predominantly acts repressively on the overall miRNA composition [5,21-24] and we indeed observed widespread downregulation of miRNA expression during murine MYCN-driven neuroblastoma development (Fig. 4C, gray line). Nonetheless, the fraction of MYCN-targeting miRNAs that is downregulated is larger than expected by chance (Fig. 4C, black line). The mechanisms by which MYCN directly represses transcription are not fully understood. Several lines of evidence suggest that it acts in a protein complex including SP1 and/or MIZ1 [25-29] to recruit histone writers [25,27-30] and/or DNA-methyltransferases $[26,30]$ to the promoter regions of its repressed target genes. Whether binding of MYCN to the canonical E-box sequence is required for this process is still unclear [26,31]. Notably, only two MYCNtargeting miRNAs in neuroblastoma (miR-29a-3p and miR-29c-3p) are directly downregulated upon induction of MYCN in a neuroblastoma cell line model, suggesting that downregulation of MYCN-targeting miRNAs during

\section{SHEP-MYCN-ER}

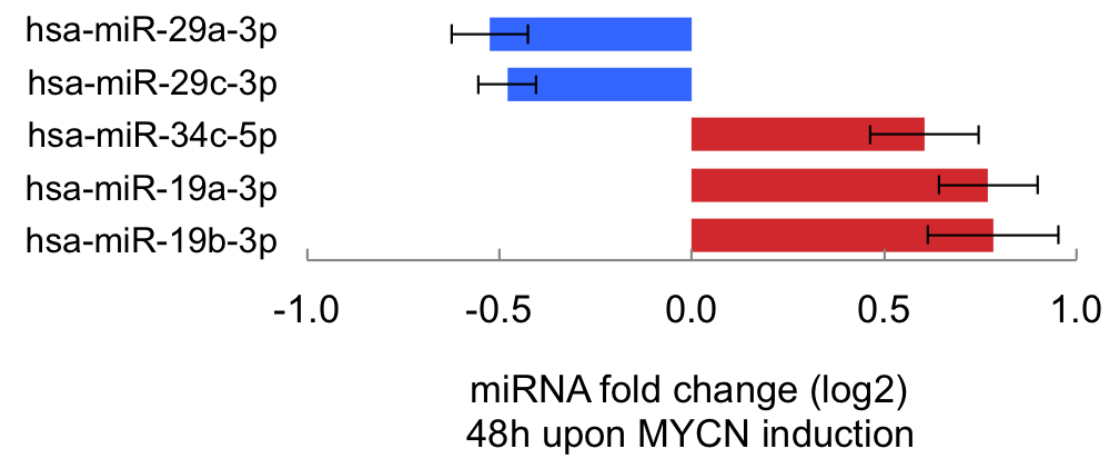

Figure 5: Induction of MYCN activity is associated with altered expression levels of particular miRNAs that target its 3'UTR. Summary of significantly differentially expressed miRNAs upon MYCN induction relative to control treatment (Student t-test, Benjamini \& Hochberg multiple testing corrected p-value $<0.05$ ). Data are represented in a horizontal bar graph as log 2 fold changes \pm standard error of the mean (5 biological replicates). 
tumor formation is likely to result from secondary (epi) genetic events downstream of increased MYCN activity.

The integration of the screen results with miRNA expression data from MYCN non-amplified primary neuroblastoma tumors further identified $5 \mathrm{MYCN}$ targeting miRNAs with positive correlation to MYCN expression or activity. Subsequent analysis of these miRNAs in in vivo MYCN model systems showed that activation of $\mathrm{MYCN}$ is associated to increased expression of miR-19a-3p, miR-19b-3p and miR-494-3p. Indeed, miR-19a-3p and miR-19b-3p, members of the oncogenic miR-17-92 cluster, have been shown to be direct transcriptional targets of $\mathrm{MYCN}$ in neuroblastoma cells $[5,24]$, and the region upstream to $\mathrm{miR}-494-3 \mathrm{p}$ contains E-box sequences (data not shown), suggesting that MYCN can induce its expression. It is conceivable that these miRNAs act both in a MYCN negative regulatory feed-back loop as well as via repression of important neuroblastoma tumor suppressor genes: indeed, miR19a-3p targets ESR1 [32], a ligand-inducible transcription factor implicated in neuronal differentiation, whereas miR-19b-3p represses DKK3 expression [33], a marker of tumor differentiation with elevated expression levels in favorable tumors. The MYCN model systems, however, cannot confirm the positive correlation between miR$34 c-5 p$ and miR-449a and MYCN expression or activity observed in primary neuroblastoma tumors: miR-34c$5 \mathrm{p}$ is consistently downregulated in the murine models, whereas the expression of miR-449a is not altered. Furthermore, there is no evidence that MYCN could induce the expression of these miRNAs directly. Although the expression of miR-34c-5p increases upon MYCN activation in the in vitro SHEP-MYCN-ER model system, the upstream region of miR-34c-5p does not contain E-box sequences (data not shown), and miR-449a, residing in the second intron of $C D C 20 B$, was not identified by Shohet and colleagues [24] in their screen for intronic miRNAs regulated by MYCN. Nevertheless, the different relation between $M Y C N$ and $m i R-34 c-5 p$ expression in $M Y C N$ non-amplified versus amplified environment should not be surprising: activation of miR-34c-5p, either direct or indirect, could be one of the mechanisms through which normal physiological levels of MYCN affect apoptosis. In cases where MYCN exceeds its physiological levels and oncogene-induced apoptosis is triggered, a transforming cell will need to circumvent MYCN-mediated apoptosis, for instance via downregulation of miR-34c-5p, which seems to occur in TH-MYCN and LSL-MYCN;Dbh-iCre tumors.

Of further notice, 17 out of 29 identified MYCNtargeting miRNAs were considered not to be relevant for MYCN regulation in the context of neuroblastoma, due to lack of inverse correlation to MYCN expression or activity, or due to unavailable expression data. Given the tissue specific nature of miRNA function, it is not excluded that these miRNAs regulate $\mathrm{MYCN}$ in an other cellular context, such as that of normal brain development [34] and a number of childhood malignancies with demonstrated involvement of MYCN: medulloblastoma [31,35], retinoblastoma [35,36], Wilms' tumor [36,37], rhabdomyoscaroma [37,38] and T-cell acute lymphoblastic leukemia [38]. Notably, three of these excluded miRNAs, let-7e-5p, miR-101-3p and miR-202-3p, have been reported to target $\mathrm{MYCN}$ in a MYCN amplified neuroblastoma cell line [3]. This discrepancy between literature and our data could be explained by the fact that our integrative approach identifies MYCN-targeting miRNAs in neuroblastoma using gene expression data from MYCN non-amplified primary tumors. Buechner and colleagues [3] showed that let-7e-5p, miR-101-3p and miR-202-3p are able to affect MYCN expression in a MYCN amplified neuroblastoma cell line, whereas we found no evidence that they are regulating $\mathrm{MYCN}$ in MYCN non-amplified primary neuroblastoma tumors.

In conclusion, we identify $12 \mathrm{MYCN}$-targeting miRNAs in with regulatory effects on $M Y C N$ expression levels or activity in MYCN non-amplified neuroblastoma (Fig. 2A). Furthermore, we provide evidence that these miRNAs are preferentially downregulated during MYCNdriven neuroblastoma tumor formation, suggesting that MYCN negatively controls the expression of these miRNAs, and as such safeguards its own expression. Understanding this regulatory miRNAome upstream of MYCN in neuroblastoma can open new perspectives for targeting the MYCN pathway in neuroblastoma tumors, a strategy that holds great promise [39]. Moreover, the identification of additional target genes of the miRNAs in the described MYCN-miRNA interactome might prove useful in the search for novel therapeutic targets.

\section{METHODS}

\section{MYCN 3'UTR-miRNA library screen}

HEK293T cells were seeded at a density of 10 000 cells/well in 96-well plates. Twenty-four hours after seeding, cells were co-transfected with $100 \mathrm{ng}$ of a reporter vector with the wild-type MYCN 3'UTR (ENST00000281043, Ensembl release 75) cloned downstream of the Firefly luciferase gene (SwitchGear Genomics, S207230) and $20 \mathrm{ng}$ of pRL-TK control vector containing the Renilla luciferase gene (Promega) together with a library of 470 miRNA mimics $(2.5 \mathrm{pmol})$ (Ambion's Pre-miR miRNA Precursor Library - Human V3, design based on miRBase v9.2 with exclusion of hsamiR-122a). Lipid based transfections were performed using $0.4 \mu \mathrm{l}$ Dharmafect Duo reagent (Dharmacon). Forty-eight hours post-transfection, luciferase reporter gene activities were assayed using the Dual-Luciferase Reporter assay system (Promega) according to the 
manufacturer's protocol with minor changes (LARII and Stop \& Glo buffer volumes were reduced to $50 \mu 1)$. Firefly reporter gene activities were normalized to Renilla values and then log-transformed. Subsequently, robust z-scores were calculated and median centered to the distribution of robust Z-scores from 36 analogous screens (of 17 selected cancer and disease associated genes) on a per miRNA basis in order to remove potential systematic bias. The resulting interaction scores are thus more negative for miRNAs that interact with the 3'UTR. In order to determine the interaction score cutoff that separates interactions from non-interactions, the scores for a set of validated miRNA interactions, probed in the MYCN screen or any of the analogous screens, were used together with the scores for a set of negative control interactions from an empty3'UTR vector miRNA library screen to perform ROCcurve analysis and determine the point of highest accuracy (interaction score $=-1.94$, specificity $=99 \%$, sensitivity $=51 \%$ ). MYCN 3'UTR-miRNA library screen results were replicated in two independent experiments. For a more detailed description of the 3'UTR-miRNA library screen setup and data-analysis we refer to Van Peer et al. (in preparation).

\section{miRNA target analysis}

Potential miRNA target sites in the MYCN 3'UTR were identified as reported previously [40]. In addition, miRNAs targeting MYCN were predicted using the MirTarget2 algorithm [41].

\section{miRNA and mRNA expression in patient cohort}

160 MYCN non-amplified primary tumor samples of neuroblastoma patients were collected prior to therapy at the Ghent University Hospital (Ghent, Belgium), the University Children's Hospital Essen (Essen, Germany), the Hospital Clínico Universitario (Valencia, Spain), the Academic Medical Center (University of Amsterdam, Netherlands) and the National Children's Research Centre (Dublin, Ireland). Informed consent was obtained from the patients' relatives. mRNA data from 75 primary neuroblastoma tumors is available at the Gene Expression Omnibus (http://www.ncbi.nlm.nih.gov/geo; Accession Number: GSE32664). Correlation of miRNA expression levels and MYCN mRNA levels was evaluated with Spearman's rank correlation coefficient. In order to find miRNAs that show negative correlation to MYCN activity and hence MYCN protein levels, mRNAs were ranked according to decreasing Spearman's rank correlation coefficient for each miRNA. Ranked gene lists were used for Gene Set Enrichment Analysis (GSEA) [11] with selected gene sets of $\mathrm{MYC}(\mathrm{N})$ regulated genes from the chemical and genetic perturbations gene set collections in the GSEA Molecular Signatures Database. From all gene sets in this collection that have either $M Y C$ or $M Y C N$ in their name, the six gene sets that contain MYC(N) upregulated genes were used for this analysis: COLLER MYC TARGETS UP [12], DANG MYC TARGETS UP [13], DANG REGULATED BY MYC UP [13], KIM MYC AMPLIFICATION TARGETS UP [14], LEE LIVER CANCER MYC UP [42], SCHUHMACHER MYC TARGETS UP [16] and YU MYC TARGETS UP [17]. Gene sets with a false discovery rate (FDR) below $25 \%$ and a normalized enrichment score (NES) below -2 were considered significantly negatively enriched.

\section{miRNA annotation}

In this study we used data obtained with three different analytical miRNA platforms. Platform designs are based on different releases of the miRBase database for miRNA annotation, resulting in non-overlapping annotation of data sets. To allow for correct integration of miRNA data, we used miRBase Tracker, an in-house developed web tool for miRNA reannotation [43] and applied the most up-to-date annotation at time of publication (miRBase release 21). Details of reannotation of the different miRNA platforms, including cross-species comparison, can be found in Supplementary Tables S1 S5.

\section{SHEP-MYCN-ER model system}

The MYCN-inducible SHEP-MYCN-ER cell model [44] was kindly provided by Johannes H Schulte (Department of Pediatric Oncology and Hematology, University Children's Hospital Essen, Germany). Cell line authenticity was validated by Short Tandem Repeat (STR) genotyping prior to performing the described experiments. In the SHEP-MYCN-ER cell line, the cDNA of MYCN is fused to a mutated estrogen responsive domain (ER) which can bind 4-hydroxy-tamoxifen (4-OHT), but is unable to bind with natural estrogen [44]. Addition of 4-OHT to the culture medium (200 nM final concentration) activates the MYCN-ER expression. SHEP-MYCN-ER cells were seeded at 100000 cells per 6-well and treated with 4-OHT after $48 \mathrm{~h}$. Subsequently, cells were pelleted for RNA isolation $48 \mathrm{~h}$ after treatment. In parallel, cells were treated with equal amounts of the 4-OHT solvent (70\% ethanol) as a negative control. RNA was isolated using the miRNeasy Mini Kit (Qiagen; 217004) according to manufacturer's instructions. Five replicate experiments were performed and analyzed.

\section{RT-qPCR}

RT-qPCR reactions were performed and reported according to the MIQE guidelines [45]. For quantification 
of individual miRNA expressions, cDNA was synthesized from $500 \mathrm{ng}$ total RNA with $4 \mu \mathrm{l}$ of HiSpec Buffer, $2 \mu \mathrm{l}$ of Nucleics Mix and $2 \mu \mathrm{l}$ miScript RT Mix (miScript II RT Kit, Qiagen; 218161) in a final volume of $20 \mu \mathrm{l}$. This reaction mix was incubated for $60^{\prime}$ at $37^{\circ} \mathrm{C}$ and $5^{\prime}$ at $95^{\circ} \mathrm{C}$ using an iCycer instrument (Bio-Rad). qPCR reactions contained $3 \mathrm{ng}$ of cDNA, $2.5 \mu \mathrm{l}$ QuantiTeckt Mastermix, $0.5 \mu \mathrm{l}$ miScript Universal Primer and $0.5 \mu \mathrm{l}$ miRNAspecific miScript Primer Assay (Qiagen, miScript Primer Assays used are listed in Supplementary Table S2) in a total volume of $5 \mu \mathrm{l}$. Expression levels were normalized against three stably expressed reference miRNAs (hsamiR-125a, hsa-miR-423 and hsa-miR-92) validated with GeNorm [46] and analyzed using qbase+ software version 2.6 (http://www.biogazelle.com/qbaseplus) [47].

\section{TH-MYCN neuroblastoma progression model}

TH-MYCN+/+ mice [18] were sacrificed at day seven $(n=4)$ and day fourteen $(n=4)$ of life to harvest sympathetic ganglia containing foci of neuroblast hyperplasia, and at week 6 of life to harvest advanced neuroblastoma tumors $(n=4)$. Additionally, we have dissected the same sympathetic ganglia from $\mathrm{TH}-$ MYCN-/- mice at day seven $(n=4)$, day fourteen $(n=4)$ and week $6(n=4)$ of life to control for miRNA expression changes during normal development. Murine total RNA was isolated using the miRNeasy Mini Kit (Qiagen, 217004) according to the manufacturer's instructions. Mature miRNA expression levels were quantified using a stem-loop RT-qPCR platform. Briefly, $99 \mathrm{ng}$ of total RNA was reverse transcribed using Megaplex ${ }^{\text {TM }}$ RT Primers, Rodent Pool Set v3.0 (Life Technologies Applied Biosystems; 4444746) followed by a 12-cycle pre-amplification according to the manufacturer's instructions. Pre-amplified cDNA was diluted four times and quantified using the TaqMan Array Rodent MicroRNA A+B Cards Set v3.0 (Life Technologies - Applied Biosystems; 4444909) according to the manufacturer's instructions on a ViiA 7 Real-Time PCR System (Life Technologies - Applied Biosystems). Only $\mathrm{C}_{\mathrm{q}}$ values lower than 32 were retained and normalized using global mean normalization, as previously described [48]. Furthermore, to allow for cross-species comparison of miRNA expression data, we only considered those murine miRNA assays that target sequences similar to the sequences of the human miRNAs included in our 3'UTR-miRNA library screen (see Supplementary Tables $\mathrm{S} 3$ - S4). Linear regression analysis was performed to evaluate the differential temporal expression pattern in ganglia from wild-type mice and ganglia and tumors from transgenic mice. A $\pi$-value metric was subsequently calculated as the difference in regression slopes between transgenic and wild-type samples, multiplied with the statistical significance of this difference: $\pi-$ value $=-\log _{10} \mathrm{p}$-value $\times \Delta_{\text {slope regression }}$

\section{LSL-MYCN;Dbh-iCre tumors}

The LSL-MYCN;Dbh-iCre mouse model is described elsewhere [20] along with details on tumor dissection and miRNA expression profiling. In brief, 60 ng of total RNA of normal adrenals and LSL-MYCN;DbhiCre tumors, isolated with the miRNeasy Mini kit (Qiagen), was used to quantify murine mature miRNA expression levels using a stemloop RT-qPCR platform (Life Technologies-Applied Biosystems).

\section{Statistical methods}

All statistical analyses were performed using $\mathrm{R}$ Bioconductor software (version 3.0.2). If not further specified in the results section, statistical significance was defined as $p$-value $<0.05$ for all statistical tests.

\section{Data accessibility}

mRNA data from 75 primary neuroblastoma tumors are available at the Gene Expression Omnibus (http:// www.ncbi.nlm.nih.gov/geo) under accession number GSE32664. miRNA expression data from the TH-MYCN neuroblastoma progression model are available in the ArrayExpress database (www.ebi.ac.uk/arrayexpress) under accession number E-MTAB-2618. Furthermore, the processed data can be visualized via the R2 microarray analysis and visualization platform (http://r2.amc.nl) under experiment "Exp Nb Hyperplasia TH-MYCN - Ghent - 24 - custom - mirbase19mm2”.

\section{ACKNOWLEDGMENTS}

We would like to thank the Ghent University Hospital (Ghent, Belgium), the University Children's Hospital Essen (Essen, Germany) and the Hospital Clínico Universitario (Valencia, Spain) for collection of primary tumor samples. Further, we would like to thank F. De Vloed for excellent technical support and J.J.B. Koster for loading the murine miRNA expression data and accompanying statistics in R2.

\section{Grant support}

This work was supported by the GOA (grant number 01G01910), by research grants from the European Union (FP7-ASSET project) to F.S., from the Belgian Foundation against Cancer (Stichting Tegen Kanker) to J.V. [SCIE 2010-177], and from the Fund for Scientific 
Research Flanders (FWO) to F.S. [G.0530.12N]; by a $\mathrm{PhD}$ grant from the Agency for Innovation by Science and Technology (IWT) to A.B. [IWT 101506]; an Emmanuel van der Schueren research grant from the Flemish League against Cancer (Vlaamse Liga tegen Kanker) to G.V.P.; a $\mathrm{PhD}$ grant from the Ghent University to G.V.P. [BOF 01D35609]; and a post-doc grant of the FWO to P.M. and K.D.P. This work was further supported by Program Grants from the NHMRC Australia, the Cancer Institute NSW and the Cancer Council NSW to D.C., B.C. and G.M..

\section{Disclosure of potential conflicts of interest}

No potential conflicts of interest are disclosed

\section{Supplementary data}

Supplementary Data include Supplementary Tables S1-S7 and Supplementary Figures S1-S3.

\section{REFERENCES}

1. Meyer N, Penn LZ. Reflecting on 25 years with MYC. Nat Rev Cancer. 2008; 8: 976-990.

2. Molenaar JJ, Domingo-Fernández R, Ebus ME, Lindner S, Koster J, Drabek K, Mestdagh P, van Sluis P, Valentijn LJ, van Nes J, Broekmans M, Haneveld F, Volckmann R, Bray I, Heukamp L, et al. LIN28B induces neuroblastoma and enhances MYCN levels via let-7 suppression. Nat Genet. 2012; 44: 1199-1206.

3. Buechner J, Tomte E, Haug BH, Henriksen JR, Løkke C, Flaegstad T, Einvik C. Tumour-suppressor microRNAs let7 and mir-101 target the proto-oncogene MYCN and inhibit cell proliferation in MYCN-amplified neuroblastoma. British Journal of Cancer. 2011; 105: 296-303.

4. Peukert K, Staller P, Schneider A, Carmichael G, Hänel F, Eilers M. An alternative pathway for gene regulation by Myc. The EMBO Journal. 1997; 16: 5672-5686.

5. Mestdagh P, Fredlund E, Pattyn F, Schulte JH, Muth D, Vermeulen J, Kumps C, Schlierf S, De Preter K, Van Roy N, Noguera R, Laureys G, Schramm A, Eggert A, Westermann F, et al. MYCN/c-MYC-induced microRNAs repress coding gene networks associated with poor outcome in MYCN/c-MYC-activated tumors. Oncogene. 2010; 29: 1394-1404.

6. Ramaswami R, Harding V, Newsom-Davis T. Novel cancer therapies: treatments driven by tumour biology. Postgrad Med J. 2013; 89: 652-658.

7. Di Leva G, Garofalo M, Croce CM. MicroRNAs in cancer. Annu Rev Pathol. 2014; 9: 287-314.

8. Wei JS, Song YK, Durinck S, Chen Q-R, Cheuk ATC, Tsang P, Zhang Q, Thiele CJ, Slack A, Shohet J, Khan J. The MYCN oncogene is a direct target of miR-34a.
Oncogene. 2008; 27: 5204-5213.

9. Mestdagh P, Lefever S, Pattyn F, Ridzon D, Fredlund E, Fieuw A, Ongenaert M, Vermeulen J, De Paepe A, Wong L, Speleman F, Chen C, Vandesompele J. The microRNA body map: dissecting microRNA function through integrative genomics. Nucleic Acids Research. 2011; 39: e136.

10. Fontana L, Fiori ME, Albini S, Cifaldi L, Giovinazzi $\mathrm{S}$, Forloni M, Boldrini R, Donfrancesco A, Federici V, Giacomini P, Peschle C, Fruci D. Antagomir-17-5p Abolishes the Growth of Therapy-Resistant Neuroblastoma through p21 and BIM. Maas S, editor. PLoS ONE. 2008; 3 : e2236.

11. Subramanian A, Tamayo P, Mootha VK, Mukherjee S, Ebert BL, Gillette MA, Paulovich A, Pomeroy SL, Golub TR, Lander ES, Mesirov JP. Gene set enrichment analysis: a knowledge-based approach for interpreting genome-wide expression profiles. Proceedings of the National Academy of Sciences of the United States of America. 2005; 102: $15545-15550$.

12. Coller HA, Grandori C, Tamayo P, Colbert T, Lander ES, Eisenman RN, Golub TR. Expression analysis with oligonucleotide microarrays reveals that MYC regulates genes involved in growth, cell cycle, signaling, and adhesion. Proceedings of the National Academy of Sciences of the United States of America. 2000; 97: 3260-3265.

13. Zeller KI, Jegga AG, Aronow BJ, O’Donnell KA, Dang $\mathrm{CV}$. An integrated database of genes responsive to the Myc oncogenic transcription factor: identification of direct genomic targets. Genome Biology. 2002; 4: R69-R69.

14. Kim J, Woo AJ, Chu J, Snow JW, Fujiwara Y, Kim CG, Cantor AB, Orkin SH. A Myc network accounts for similarities between embryonic stem and cancer cell transcription programs. Cell. 2010; 143: 313-324.

15. Lee RC. An Extensive Class of Small RNAs in Caenorhabditis elegans. Science. 2001; 294: 862-864.

16. Schuhmacher M, Kohlhuber F, Hölzel M, Kaiser C, Burtscher H, Jarsch M, Bornkamn G, Laux G, Polack A, Weidle U, Eick D. The transcriptional program of a human B cell line in response to Myc. Nucleic Acids Research. 2001; 29: 397-406.

17. $\mathrm{Yu}$ D, COZMA D, PARK A, Thomas-Tikhonenko A. Functional validation of genes implicated in lymphomagenesis: an in vivo selection assay using a Mycinduced B-cell tumor. Ann N Y Acad Sci. 2005; 1059: $145-159$.

18. Weiss WA, Aldape K, Mohapatra G, Feuerstein BG, Bishop JM. Targeted expression of MYCN causes neuroblastoma in transgenic mice. The EMBO Journal. 1997; 16: 29852995.

19. Hansford LM, Thomas WD, Keating JM, Burkhart CA, Peaston AE, Norris MD, Haber M, Armati PJ, Weiss WA, Marshall GM. Mechanisms of embryonal tumor initiation: distinct roles for $\mathrm{MycN}$ expression and $\mathrm{MYCN}$ 
amplification. Proceedings of the National Academy of Sciences of the United States of America. 2004; 101: 12664-12669.

20. Althoff K, Beckers A, Bell E, Nortmeyer M, Thor T, Sprüssel A, Lindner S, De Preter K, Florin A, Heukamp LC, Klein-Hitpass L, Astrahantseff K, Kumps C, Speleman F, Eggert A, et al. A Cre-conditional MYCN-driven neuroblastoma mouse model as an improved tool for preclinical studies. Oncogene. 2014.

21. Chen Y, Stallings RL. Differential Patterns of MicroRNA Expression in Neuroblastoma Are Correlated with Prognosis, Differentiation, and Apoptosis. Cancer Research. 2007; 67: 976-983.

22. Bray I, Bryan K, Prenter S, Buckley PG, Foley NH, Murphy DM, Alcock L, Mestdagh P, Vandesompele J, Speleman F, London WB, McGrady PW, Higgins DG, O'Meara A, O'Sullivan M, et al. Widespread dysregulation of MiRNAs by MYCN amplification and chromosomal imbalances in neuroblastoma: association of miRNA expression with survival. PLoS ONE. 2009; 4: e7850.

23. Schulte JH, Schowe B, Mestdagh P, Kaderali L, Kalaghatgi P, Schlierf S, Vermeulen J, Brockmeyer B, Pajtler K, Thor T, De Preter K, Speleman F, Morik K, Eggert A, Vandesompele $\mathrm{J}$, et al. Accurate prediction of neuroblastoma outcome based on miRNA expression profiles. Int J Cancer. 2010; 127: 2374-2385.

24. Shohet JM, Ghosh R, Coarfa C, Ludwig A, Benham A, chen Z, Patterson DM, Barbieri E, Mestdagh P, Sikorski DN, Milosavljevic A, Kim ES, Gunaratne PH. A genomewide search for promoters that respond to increased MYCN reveals both new oncogenic and tumor suppressor microRNAs associated with aggressive neuroblastoma. Cancer Research. 2011; 71: 3841-3851.

25. Liu T, Tee AEL, Porro A, Smith SA, Dwarte T, Liu PY, Iraci N, Sekyere E, Haber M, Norris MD, Diolaiti D, Valle Della G, Perini G, Marshall GM. Activation of tissue transglutaminase transcription by histone deacetylase inhibition as a therapeutic approach for Myc oncogenesis. Proceedings of the National Academy of Sciences of the United States of America. 2007; 104: 18682-18687.

26. Gherardi S, Valli E, Erriquez D, Perini G. MYCN-mediated transcriptional repression in neuroblastoma: the other side of the coin. Front Oncol. 2013; 3: 42.

27. Iraci N, Diolaiti D, Papa A, Porro A, Valli E, Gherardi S, Herold S, Eilers M, Bernardoni R, Valle Della G, Perini G. A SP1/MIZ1/MYCN repression complex recruits HDAC1 at the TRKA and p75NTR promoters and affects neuroblastoma malignancy by inhibiting the cell response to NGF. Cancer Research. 2011; 71: 404-412.

28. Lodrini M, Oehme I, Schroeder C, Milde T, Schier MC, Kopp-Schneider A, Schulte JH, Fischer M, De Preter K, Pattyn F, Castoldi M, Muckenthaler MU, Kulozik AE, Westermann F, Witt O, et al. MYCN and HDAC2 cooperate to repress miR-183 signaling in neuroblastoma. Nucleic Acids Research. 2013; 41: 6018-6033.
29. Corvetta D, Chayka O, Gherardi S, D'Acunto CW, Cantilena S, Valli E, Piotrowska I, Perini G, Sala A. Physical interaction between $\mathrm{MYCN}$ oncogene and polycomb repressive complex 2 (PRC2) in neuroblastoma: functional and therapeutic implications. Journal of Biological Chemistry. 2013; 288: 8332-8341.

30. Brenner C, Deplus R, Didelot C, Loriot A, Viré E, De Smet C, Gutierrez A, Danovi D, Bernard D, Boon T, Pelicci PG, Amati B, Kouzarides T, de Launoit Y, Di Croce L, et al. Myc represses transcription through recruitment of DNA methyltransferase corepressor. The EMBO Journal. 2005; 24: 336-346.

31. Roussel MF, Robinson GW. Role of MYC in Medulloblastoma. Cold Spring Harb Perspect Med. 2013; 3.

32. Lovén J, Zinin N, Wahlström T, Müller I, Brodin P, Fredlund E, Ribacke U, Pivarcsi A, Påhlman S, Henriksson M. MYCN-regulated microRNAs repress estrogen receptoralpha (ESR1) expression and neuronal differentiation in human neuroblastoma. Proceedings of the National Academy of Sciences of the United States of America. 2010; 107: 1553-1558.

33. De Brouwer S, Mestdagh P, Lambertz I, Pattyn F, De Paepe A, Westermann F, Schroeder C, Schulte JH, Schramm A, De Preter K, Vandesompele J, Speleman F. Dickkopf-3 is regulated by the MYCN-induced miR-17-92 cluster in neuroblastoma. Int J Cancer. 2011.

34. Hurlin PJ. N-myc functions in transcription and development. Birth Defect Res C. 2005; 75: 340-352.

35. Rushlow DE, Mol BM, Kennett JY, Yee S, Pajovic S, Thériault BL, Prigoda-Lee NL, Spencer C, Dimaras H, Corson TW, Pang R, Massey C, Godbout R, Jiang Z, Zacksenhaus $\mathrm{E}$, et al. Characterisation of retinoblastomas without RB1 mutations: genomic, gene expression, and clinical studies. Lancet Oncol. 2013; 14: 327-334.

36. Nisen PD, Zimmerman KA, Cotter SV, Gilbert F, Alt FW. Enhanced expression of the N-myc gene in Wilms' tumors. Cancer Research. 1986; 46: 6217-6222.

37. Driman D, Thorner PS, Greenberg ML, Chilton-MacNeill S, Squire J. MYCN gene amplification in rhabdomyosarcoma. Cancer. 1994; 73: 2231-2237.

38. Astolfi A, Vendemini F, Urbini M, Melchionda F, Masetti R, Franzoni M, Libri V, Serravalle S, Togni M, Paone G, Montemurro L, Bressanin D, Chiarini F, Martelli AM, Tonelli R, et al. MYCN is a novel oncogenic target in pediatric T-cell acute lymphoblastic leukemia. Oncotarget. 2014; 5: 120-130.

39. Barone G, Anderson J, Pearson ADJ, Petrie K, Chesler L. New strategies in neuroblastoma: Therapeutic targeting of MYCN and ALK. Clin Cancer Res. 2013; 19: 5814-5821.

40. Grimson A, Farh KK-H, Johnston WK, Garrett-Engele P, Lim LP, Bartel DP. MicroRNA targeting specificity in mammals: determinants beyond seed pairing. Molecular Cell. 2007; 27: 91-105.

41. Wang X, Naqa El IM. Prediction of both conserved 
and nonconserved microRNA targets in animals. Bioinformatics. 2008; 24: 325-332.

42. Lee J-S, Chu I-S, Mikaelyan A, Calvisi DF, Heo J, Reddy JK, Thorgeirsson SS. Application of comparative functional genomics to identify best-fit mouse models to study human cancer. Nat Genet. 2004; 36: 1306-1311.

43. Van Peer G, Lefever S, Anckaert J, Beckers A, Rihani A, Van Goethem A, Volders P-J, Zeka F, Ongenaert M, Mestdagh P, Vandesompele J. miRBase Tracker: keeping track of microRNA annotation changes. Database. Oxford University Press; 2014; 2014: bau080-bau080.

44. Schulte JH, Horn S, Otto T, Samans B, Heukamp LC, Eilers U-C, Krause M, Astrahantseff K, Klein-Hitpass L, Buettner R, Schramm A, Christiansen H, Eilers M, Eggert A, Berwanger B. MYCN regulates oncogenic MicroRNAs in neuroblastoma. Int J Cancer. 2008; 122: 699-704.

45. Bustin SA, Benes V, Garson JA, Hellemans J, Huggett J, Kubista M, Mueller R, Nolan T, Pfaffl MW, Shipley GL, Vandesompele J, Wittwer CT. The MIQE guidelines: minimum information for publication of quantitative realtime PCR experiments. Clinical chemistry. 2009. pp. 611622.

46. Vandesompele J, De Preter K, Pattyn F, Poppe B, Van Roy N, De Paepe A, Speleman F. Accurate normalization of real-time quantitative RT-PCR data by geometric averaging of multiple internal control genes. Genome Biology. 2002; 3: R34.

47. Hellemans J, Mortier G, De Paepe A, Speleman F, Vandesompele J. qBase relative quantification framework and software for management and automated analysis of real-time quantitative PCR data. Genome Biology. 2007; 8: R19.

48. Mestdagh P, Van Vlierberghe P, De Weer A, Muth D, Westermann F, Speleman F, Vandesompele J. A novel and universal method for microRNA RT-qPCR data normalization. Genome Biology. 2009; 10: R64. 